BNWL-156

\title{
THE STATIONARY CALCULATION OF RATIOS IN REACTOR STATICS
}

REPORT

JEFFERY LEWINS

OCTOBER, 1965

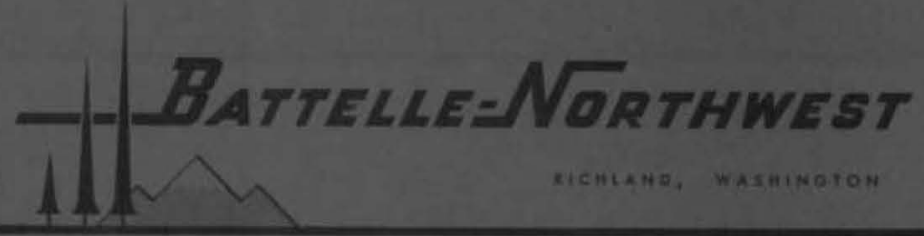

PACIFIC NORTHWEST LABORATORY operated by BATTELLE MEMORIAL INSTITUTE 


\section{LEGAL NOTICE}

This report was prepared as an account of Government sponsored work. Neither the United States, nor the Commission, nor any person acting on behalf of the Commission:

A. Makes any warranty or representation, expressed or implied, with respect to the occuracy, completeness, or usefulness of the information contoined in this report, or that the use of any information, apparatus, method, or process disclosed in this report may not infringe privately owned rights; or

B. Assumes any liobilities with respect to the use of, or for damages resulting from the use of ony information, apparatus, method, or process disclosed in this report.

As used in the obove, "person acting on behalf of the Commission" includes any emplayee or contractor of the Commission, or employee of such contractor, to the extent that such employee or con. fractor of the Commission, or employee of such contractor prepores, disseminotes, or provides occess to, any information pursuant to his employment or contract with the Commission, or his employment with such contractor.

\section{PACIFIC NORTHWEST LABORATORY}

RICHLAND, WASHINGTON

operated by

BATTELLE MEMORIAL INSTITUTE

for the

UNITED STATES ATOMIC ENERGY COMMISSION UNDER CONTRACT AT(45-1)-1830 
Title: The Stationary Calculation of Ratios in Reactor Statics Author: J. Lewins

Date: October, 1965

UNCLASSIFIED

Page 2, line 7: alone. Therefore

Page 4, paragraph 3: we have drawn on Pomranning

Page 7,1 ine $6:$ The general procedure is to add, to the characteristic of interest, an integral over the equations to be satisfied...

Page 9, paragraph 3: The remaining production processes

Page 10, Eq. (23): for $N^{2}$ read $\stackrel{\sim}{N}$

Page 13, line 1: $\quad\left(\mathrm{N}_{0}^{+}\right)$

$\mathrm{Eq} \cdot(30): \lambda_{\mathrm{q}}$

Eq. (31): $=F^{*} N^{2}+(j)$

Page 16, Eq. (42): $=\mathrm{H}^{+}-\mathrm{F}^{*} \tilde{N}^{+(\infty)}+\mathrm{F}^{*} \mathrm{C}^{+}$

Page 18, Eq. (49): $F^{*} \hat{N}(j+1) d x$ 
BNWL -156

UC-34, Physics
FIRST
UNRESTRICTED
DISTRIBUTION
MADE

THE STATIONARY CALCULATION

OF RATIOS IN REACTOR STATICS

\author{
By \\ Jeffery Lewins* \\ Theoretical Physics Unit \\ Reactor Physics Section \\ Physics and Instrument Department
}

October, 1965

\title{
PACIFIC NORTHWEST LABORATORY RI CHLAND, WASH INGTON
}

* Major, Royal Engineers, British Army Visiting Professor of University of Washington 
Printed in USA. Price $\$ 2.00$. Available from the Clearinghouse for Federal Scientific and Technical Information, National Bureau of Standards,

U. S. Department of Commerce, Springfield, Virginia 


\title{
THE STATIONARY CALCULATION \\ OF RATIOS IN REACTOR STATICS
}

\begin{abstract}
$\underline{\text { ABSTRACT }}$
Expressions are given for the stationary calculations of arbitrary ratios of interest in critical systems and in steady state, sub-critical systems having a source. Emphasis is placed on the calculation of the adjoint functions by iterative methods already used in standard reactor codes and on the reduction in computing time offered by stationary methods compared to direct computation. Both variational principles and perturbation expressions are provided.
\end{abstract}




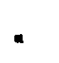




\section{THE STATIONARY CALCULATION \\ DF RATIOS IN REACTOR STATICS}

INTRODUCTION

The effective multiplication factor is a primary characteristic of interest in source free systems. More mathematically, one studies the eigenvalue of such a system in the form of a linear multiplier of the production term in the neutron balance equation. Yet, there are other characteristics of interest, to be computed in critical systems or in steady state, sub-critical systems having a source. An interesting class of such characteristics is arbitrary ratios.

Some examples of arbitrary* ratios are

- Breeding ratios

- Peak to average power ratios

- Thermal utilization

- Resonance escape probability

- Neutron lifetime.

Indeed, in a critical system with arbitrary normalization or power, any reaction of interest can only be evaluated in terms of a ratio to the power level or flux.

This report discusses ways to calculate ratios in a stationary fashion such that the precision in the ratio is greater than the precision to which we know the flux or neutron density. It does so for both variational methods (where the ratio is calculated ab initio) and for perturbation methods (where the change in the ratio is to be found due to a perturbation in the system properties). The impetus for this account comes from Usachev's

* By arbitrary, we mean that the ratio depends on the weighting function selected in the integrals of the numerator and denominator of the ratio. This differs from the eigenvalue (or effective multiplication) which is a ratio of production to removal that is defined to be the same at every point in the reactor and hence is independent of any averaging or weighting function. 
study of the iterated transport equation in a perturbation theory for ratios. (1) In setting up the stationary calculation, we shall introduce two adjoint functions (as compared with the one adjoint function of conventional stationary methods for the eigenvalue or effective multiplication). At first glance, this appears to triple the computational labor compared to a calculation of the flux alone therefore, a few words on the raison d'être are in order. Of course, it is true that if the flux or density equations are solved exactly, any ratio of interest can be found exactly by a simple quadrature over the known flux. But it would be naive to suppose that an exact flux could ever be computed. At some point in a realistic numerical computation (particularly the widely used iterative solution), a decision has to be made to accept any remaining inaccuracies.

Naturally, the remaining error is not known, but one may examine the convergence, by which we mean the difference between successive solutions. If this is small, it could indicate that we are close to the correct solution, or it might only indicate a poor method of solution! We can set a criterion of convergence for the flux and accept the solution when the criterion is satisfied throughout the system.

The error and, therefore, the convergence tend to fall exponentially so that to halve the convergence is likely to take much more than twice the iterations. A stationary calculation allows the convergence criterion to be relaxed while still leading to the same accuracy in the ultimate characteristic of interest. Typical experience with variation-iteration calculations in eigenvalue problems suggests that despite having to calculate an adjoint as well as a density function, net savings of a factor of 10 are realizable for the same accuracy in the effective multiplication. Similar economics are anticipated here.

In addition, the apparent factor of 3 to compute three solutions in the stationary calculation can be reduced by clever 
programming. Much of the data needed to compute solutions to flux and adjoint equations is common to both, i.e., the properties of a region. If the solutions are carried on simultaneously, repetition of the more tedious elements of the calculation can be eliminated. Thus, the multigroup diffusion adjoint equations call for a transposition of terms appearing in the flux equations; reordering the adjoint groups makes the terms common. Experience with the transport theory $\mathrm{s}-\mathrm{code}^{(2)}$ for eigenvalue problems suggests that simultaneous computation of an adjoint function increases the total required time by on $1 y$ 25\%. Hopefully, two adjoint functions will require an increase of $50 \%$ (not $300 \%$ ) over the time to compute the flux to the same accuracy.

Finally, in computing ratios and changes in ratios in multiplying systems, we are commonly dealing with the difference between nearly equal quantities; their direct calculation to sufficient accuracy is tedious. In a perturbation problem, each perturbed equation must be solved to considerable accuracy, avoiding round off error--the direct first order approximation being wildly inaccurate. Special cases arise, therefore, where the perturbation method offers extreme advantages. Consider the problem of computing the uncertainty in the breeding ratio due to uncertainties in the cross-sections used. For example, we may have five materials, each represented by a 16 group crosssection library. The direct computation calls for 80 solutions to the flux equation, and each solution must be of acceptable precision. In contrast, the perturbation method calls only for the three solutions: the flux and two adjoint functions. The 80 perturbations in the ratio are then obtained by simple quadrature over the cross-section perturbations. A11 is not rosy and a few caveats are in order. Is the ratio, to be calculated, of really direct interest? If it is to be used subsequently as an element on $1 y$ of some other computation (e.g., the neutron 
lifetime in a calculation of reactor period), there may be little point in calculating these elements separately, when one calculation would determine the characteristic of true interest.

Furthermore, as is basic to the stationary method, only one "best" calculation is available at a time. If a different ratio is wanted, a new computation of the adjoint functions is needed. Correspondingly, a flux solution of accuracy adequate for a stationary calculation is likely to be inaccurate if utilized for any direct calculation.

In the next section we derive the stationary expressions and the adjoint equations or stationary conditions. The final section discusses methods of solution for the adjoint equations. A detailed physical account of the importance has been given elsewhere. (3)

In addition to Usachev's work, $(1,4)$ we have selected Pomranning (unpublished) for a general variational principle and Selengut (unpublished) for inhomogeneous perturbation theory; this particular application to the calculation of ratios is a specialization of their discussions. STEADY STATE SUBCRITICAL SYSTEMS'

We use a compact notation encompassing the usual models of reactor statics, writing a steady state neutron density equation in the form:

$$
M N+S=0 .
$$

Here $M(x)$ is a net multiplication operator; $N(x)$, a density; and $S(x)$, an independent steady source distribution. The variable $x$ is to describe both position and velocity, to the extent called for by the model selected. In a group representation of the energy, $N$ and $S$ would be column vectors and $M$, a matrix of operators.

We also consider adjoint functions of the same $x$ variables, such as $\mathrm{N}^{+}(\mathrm{x}), \mathrm{C}^{+}(\mathrm{x})$ etc. These satisfy boundary conditions, 
analogous to the density boundary conditions, selected by their convenience in deriving a unique adjoint operator for every operator in the density equation. Thus, if $F$ is an operator, its adjoint, $F^{*}$, is defined by a commutation relation,

$$
\int \mathrm{NF}^{*} \psi^{+} \mathrm{dx}=\int \psi^{+} \mathrm{FNdx}
$$

Here $\psi^{+}$is any adjoint function satisfying the adjoint boundary conditions; $N$, any density function satisfying density boundary conditions. The integral is taken over the whole reactor space, position and velocity. Our convention, in a matrix and vector model, is that vectors on the right are column, on the left are row, so that these integrands are scalar or inner products. In such a case, the summation over the discrete energy intervals has been carried out by the scalar product, and only the integral over the position remains to be evaluated.

A steady state generalization of conventional variational and perturbation theory considers an adjoint equation based on an adjoint source, $\mathrm{H}^{+}(x)$, for an adjoint function, $S^{+}(x)$, thus,

$$
\mathrm{M}^{*} \mathrm{~S}^{+}+\mathrm{H}^{+}=0
$$

Suppose we wish to compute a characteristic of interest, $\overline{\mathrm{N}}=\int \mathrm{H}^{+} \mathrm{Ndx}$. We identify the weighting function in our characteristic with the adjoint source term in Equation (3). For example, if $\bar{N}$ is to be some reaction rate in the system, $\mathrm{H}^{+}(\mathrm{x})$ would be the reaction probability. If we were to work in terms of flux in place of density, $\mathrm{H}^{+}$would be a reaction cross-section. As such, it would vary with energy and with position.

The commutation relationship shows that Equations (1) and (3) lead to

$$
\overline{\mathrm{N}}=\int \mathrm{H}^{+} \mathrm{Ndx}=\int \mathrm{S}^{+} \mathrm{Sdx}
$$

so that $S^{+}(x)$ is the weighting to be given the source in an alternative calculation of $\bar{N}$. For this reason, $S^{+}(x)$ is the 
source importance, the contribution of a unit steady source at $x$ to the characteristic.

To compute a ratio $\bar{R}$ in such a system, we consider

$$
\bar{N}=\bar{R}=\frac{\int H_{n}^{+} N d x}{\int H_{d}^{+} N d x}
$$

where $\mathrm{H}_{\mathrm{n}}^{+}$(numerator) and $\mathrm{H}_{\mathrm{d}}^{+}$(denominator) are the reaction probabilities in the ratio of interest. We assume that $\mathrm{H}_{\mathrm{n}}^{+}, \mathrm{H}_{\mathrm{d}}^{+}$, and $\mathrm{M}$ are independent of the density, $N$, i.e., that we have a linear system.

For example, in computing the breeding ratio, we might take $\mathrm{H}_{\mathrm{n}}^{+}$to be proportional to the $\mathrm{U}^{238}$ capture cross-section (to breed plutonium) and $\mathrm{H}_{\mathrm{d}}^{+}$to the $\mathrm{U}^{235}$ absorption cross-section (to burnup fue 1). Computing a peak to average power ratio at a position $x^{\prime}$ calls for

$$
H_{n}^{+}(x)=\sum_{f}^{a}(x) \delta\left(x-x^{\prime}\right) ; H_{d}^{+}(x)=\frac{\sum_{f}^{a}(x)}{\int d x}
$$

where the flux $\phi=v N$ and $\delta\left(x-x^{\prime}\right)$ is the Dirac delta function. Analytic solutions with a delta function source are comparatively easy, but discrete numerical solutions are also possible. If we take

$$
\mathrm{H}^{+}=\frac{\mathrm{H}_{\mathrm{n}}^{+}}{\int \mathrm{H}_{\mathrm{d}}^{+} \mathrm{Ndx}}-\overline{\mathrm{R}} \frac{\mathrm{H}_{\mathrm{d}}^{+}}{\int \mathrm{H}_{\mathrm{d}}^{+} \mathrm{Ndx}}
$$

This does not prevent us, in a perturbation theory, from estimating the effect of feed back terms (such as fuel burnup) separately to determine perturbations in $\mathrm{M}, ~^{+}$. However, the stationary method employed here does not extend to making this separate calculation stationary. For a more general theory of non-linear systems, see Reference (3). 
for the adjoint source in Equation (4), we may construct our variational principle as

$$
\overline{\mathrm{L}}_{\mathrm{S}} \equiv \overline{\mathrm{R}}+\int \mathrm{S}^{+}[\mathrm{MN}+\mathrm{S}] \mathrm{dx} \rightarrow \overline{\mathrm{R}}
$$

where the arrow signifies "is a stationary expression for". That is, if some approximation $\tilde{N}$ is made for $N$, introducing an error $\delta N=N-N$, then $\bar{L}_{s}$ still gives a good estimate of $\bar{R}$. The general procedure is to add an integral over the equations to the characteristic of interest, to be satisfied by the density, weighted by a generalization of the Lagrange multiplier.

Clearly, if $\mathrm{N}$ is exact and satisfies equation (1), $\overline{\mathrm{L}}_{\mathrm{S}}=\overline{\mathrm{R}}$. It is necessary to show that the variation $\delta \overline{\mathrm{L}}_{\mathrm{s}}$ vanishes due to $\delta N$. We have, to first order,

$$
\delta \overline{\mathrm{L}}_{\mathrm{S}}(\delta \mathrm{N})=\frac{\int \mathrm{H}_{\mathrm{n}}^{+} \delta \mathrm{Ndx}}{\int \mathrm{H}_{\mathrm{d}}^{+} \mathrm{Ndx}}-\frac{\int \mathrm{H}_{\mathrm{n}}^{+} \mathrm{Ndx} \int \mathrm{H}_{\mathrm{d}}^{+} \delta \mathrm{Ndx}}{\left[\int \mathrm{H}_{\mathrm{d}}^{+} \mathrm{Ndx}\right]^{2}}+\int \delta \mathrm{NM}^{*} \mathrm{~S}^{+} \mathrm{dx} \text {, }
$$

but from Equation (4)

$$
\int \delta N M^{*} S^{+} d x=-\int \delta N H^{+} d x=-\frac{\int H_{n}^{+} \delta N d x}{\int H_{d}^{+} N d x}+\frac{\int H_{n}^{+} N d x \int H_{d}^{+} \delta N d x}{\int\left[H_{d}^{+} N d x\right]^{2}}
$$

so that $\delta \overline{\mathrm{L}}_{\mathrm{S}}(\delta \mathrm{N})$ vanishes to first order.

The stationary expression $\overline{\mathrm{L}}_{\mathrm{S}}$ includes terms that just offset the error introduced into a direct computation of $\bar{R}$. Apparently, no error is left but this is not really true, because first, we used a first order differential expression to evaluate the direct error in $\overrightarrow{\mathrm{R}}$ and, second, we could not compute $\mathrm{S}^{+}$exactly without a knowledge of the exact $\mathrm{N}$ appearing in the adjoint source, $\mathrm{H}^{+}$. However, making small errors in both $\mathrm{N}_{\text {and }} \mathrm{S}^{+}$will lead to a second order error in $\overline{\mathrm{L}}$ and $\overline{\mathrm{R}}$. Note that the normalization of $N$ in the adjoint source is cancelled 
when using the principles; the shape of $N$ appears only after an integration so that $\mathrm{H}^{+}$is not sensitive to the assumed density used to compute it.

From the variational principle we may obtain a perturbation expression for ratios in a noncritical system that is subject to perturbations in the weighting function, properties and source:

$$
\delta \overline{\mathrm{L}}_{\mathrm{S}}\left(\delta \mathrm{H}^{+}, \delta \mathrm{M}, \delta \mathrm{S}\right)=\int\left[\delta \mathrm{H}^{+} \mathrm{N}+\mathrm{S}^{+} \delta \mathrm{MN}+\mathrm{S}^{+} \delta \mathrm{S}\right] \mathrm{dx}
$$

where

$$
\delta \mathrm{H}^{+}=\frac{\delta \mathrm{H}_{\mathrm{n}}^{+}}{\int \mathrm{H}_{\mathrm{d}}^{+} \mathrm{Ndx}}-\overline{\mathrm{R}} \frac{\delta \mathrm{H}_{\mathrm{d}}^{+}}{\int \mathrm{H}_{\mathrm{d}}^{+} \mathrm{Ndx}} .
$$

Because the adjoint source depends on the density, even though not sensitively, it may be worthwhile to compute $\bar{R}$ from stationary expressions where adjoint sources do not have this dependence. We may do this at the expense of introducing two adjoint functions. We simply compute numerator and denominator of the ratio in separate, stationary calculations. Take two equations:

$$
\mathrm{M}^{\star} \mathrm{S}_{\mathrm{i}}^{+}+\mathrm{H}_{\mathrm{i}}^{+}=\mathrm{O} \quad \mathrm{i}=\mathrm{n}, \mathrm{d}
$$

then compute from

$$
\bar{L}_{i}=\int\left[H_{i}^{+} N+S_{i}^{+} M N+S_{i}^{+} S\right] d x \rightarrow \int H_{i}^{+} N d x
$$

or

$$
\delta \overline{\mathrm{L}}_{\mathrm{i}}\left(\delta \mathrm{H}_{\mathrm{i}}^{+}, \delta \mathrm{M}, \quad \delta \mathrm{S}\right)=\int\left[\delta \mathrm{H}_{\mathrm{i}}^{+} \mathrm{N}+\mathrm{S}_{\mathrm{i}}^{+} \delta \mathrm{MN}+\mathrm{S}_{\mathrm{i}}^{+} \delta \mathrm{S}\right] \mathrm{dx} \rightarrow \int \delta \mathrm{H}_{\mathrm{i}}^{+} \mathrm{Ndx}
$$

(see table 1, Appendix A).

STEADY STATE, CRITICAL SYSTEMS

The previous section dealt with sub critical systems maintained in a steady state by means of a source. None of the results are valid in a critical system as may easily be seen. In a critical system we have, for density and neutron importance,

$$
M N=0 ; M^{*} N^{+}=0
$$


Just as we may not introduce a source into a critical system and hope to obtain a steady state solution, so we may not introduce a positive adjoint source $\mathrm{H}^{+}$into the adjoint equations and obtain a bounded solution for the importance of, unit sources in a critical system. Clearly the flux from neutron sources increases without bounds, and this implies that the contribution of steady sources in a critical system to a characteristic of interest is unbounded. For this reason we modify the stationary procedure and again introduce two adjoint equations.

Furthermore, we shall introduce a negative adjoint source in each to offset the positive $\mathrm{H}^{+}$. With a suitable normalization of each negative source, we may hope to achieve a steady state even in a critical system.

The operator $M$ is now usefully divided into a production term $F$ (often identified with production from fission) and a removal term $R$. The remaining processes (production from scattering), if not included in $F$, are to be treated as a negative removal process. Then in a critical system

$$
\mathrm{MN}=[\mathrm{F}-\mathrm{R}] \mathrm{N}=\mathrm{O},
$$

correspondingly, the importance equation can be written

$$
\mathrm{M}^{*} \mathrm{~N}^{+}=\left[\mathrm{F}^{*}-\mathrm{R}^{*}\right] \mathrm{N}^{+}=0
$$

We now propose adjoint equations for functions $\mathrm{C}_{n}^{+}$and $\mathrm{C}_{\mathrm{d}}^{+}$of the form

$$
\mathrm{M}^{*} \mathrm{C}_{\mathrm{i}}^{+}+\mathrm{H}_{\mathrm{i}}^{+}-\mathrm{F}^{*} \mathrm{~N}_{\mathrm{i}}^{+}=0 \quad \mathrm{i}=\mathrm{n}, \mathrm{d}
$$

Here $\mathrm{N}_{\mathrm{n}}^{+}$and $\mathrm{N}_{\mathrm{d}}^{+}$are to be the importance solutions of Equation (18), but with that specific normalization that makes Equations (19) acceptable. To find this normalization, we multiply by $N(x)$ and integrate over the reactor:

$$
\int N M^{*} C_{i}^{+} d x+\int N H_{i}^{+} d x-\int N F^{*} N_{i}^{+} d x=0 \quad i=n, d \quad \text {. }
$$


By using the commutation and equation (17), the first term vanishes. Then

$$
\vec{N}_{i}=\int H_{i}^{+} N \mathrm{~d} x=\int N H_{i}^{+} \mathrm{dx}=\int N F^{*} \mathrm{H}_{i}^{+} \mathrm{dx}=\int \mathrm{H}_{i}^{+} \mathrm{FNdx} \quad \mathrm{i}=\mathrm{n}, \mathrm{d}
$$

These integrals define the necessary normalization of the otherwise known $\mathrm{N}_{i}^{+}$in a manner analogous to the Fourier coefficient for self adjoint systems. We are, in fact, filtering off the lowest mode from the adjoint source. Since the reactor is not critical in higher modes, the resulting inhomogeneous equation is acceptable. Methods of solving these equations will be discussed in the next section. Meanwhile, we give the stationary expressions for $\bar{R}$ and a change in $\bar{R}$.

The critical variational principle is

$$
\bar{L}_{c}=\frac{\int\left[H_{n}^{+}+C_{n}^{+} M\right] N d x}{\int\left[H_{d}^{+}+C_{d}^{+} M\right] N d x} \rightarrow \bar{R} \text {. }
$$

Clearly if an exact $N$ is introduced into $\bar{L}_{c}$, it yields $\bar{R}$. We must show it is also stationary to errors in $N$. We have

$$
\begin{aligned}
& \bar{L}_{c}(\tilde{N})=\bar{L}_{c}(N)+\delta \bar{L}_{c}(\delta N)=\frac{\int\left[H_{n}^{+}+C_{n}^{+} M\right] \tilde{N} d x}{\int\left[H_{d}^{+}+C_{d}^{+} M\right] N^{2} d x} \\
& =\frac{\int\left[H_{n}^{+} N+C_{n}^{+} M N\right] d x+\int \delta N\left[H_{n}^{+}+M^{*} C_{n}^{+}\right] d x}{\int\left[H_{d^{+}}^{+}+C_{d}^{+} M N\right] d x+\int \delta N\left[H_{d}^{+}+M^{*} C_{d}^{+}\right] d x} \\
& =\frac{\int H_{n}^{+} N d x+\int \delta N F^{*} N_{n}^{+} d x}{\int H_{d}^{+} N d x+\int \delta N F^{\star} N_{d}^{+} d x} \\
& =\bar{R} \frac{1+\int \delta N F^{*} N_{n}^{+} \mathrm{dx} / \int N F^{*} N_{n}^{+} \mathrm{dx}}{1+\int \delta N F^{*} N_{\mathrm{d}}^{+} \mathrm{dx} / \int N F^{*} \mathrm{~N}_{\mathrm{d}}^{+} \mathrm{dx}}=\bar{R} \text {. }
\end{aligned}
$$


In the last step, we employed the normalization of the $\mathrm{N}_{\mathrm{n}}^{+}$and $\mathrm{N}_{\mathrm{d}}^{+}$, but these two functions are the same to an arbitrary sealing factor. It follows that the integrals are proportional to each other and cancel, to leave $\bar{R}$.

It might appear that $\bar{L}=\bar{R}$ for any $N$. This again might be true if the adjoint functions were computed exactly, which is not feasible since $N$ would be needed to compute the adjoint source. knowing $\mathrm{N}$ exactly, we would not need a stationary expression. The result of simultaneous errors in $N$ and in the $\mathrm{C}_{i}^{+}$leads to an error in $\bar{R}$ of small order: $\delta \bar{L}_{C}(\delta N)=0$.

The corresponding first order perturbation theory is again easily obtained from $\bar{L}_{C}$. By construction, $N$ and the $\mathrm{C}_{i}^{+}$can be considered unperturbed, to first order, and in a logarithmic form

$\frac{\delta \overline{\mathrm{L}}_{C}\left(\delta \mathrm{H}^{+}, \delta \mathrm{M}\right)}{\overline{\mathrm{L}}_{\mathrm{C}}}=\frac{\int\left[\delta \mathrm{H}_{\mathrm{n}}^{+}+\mathrm{C}_{\mathrm{n}}^{+} \delta \mathrm{M}\right] \mathrm{Ndx}}{\int \mathrm{H}_{\mathrm{n}}^{+} \mathrm{Ndx}}-\frac{\int\left[\delta \mathrm{H}_{\mathrm{d}}^{+}+\mathrm{C}_{\mathrm{d}}^{+} \delta \mathrm{M}\right] \mathrm{Ndx}}{\int \mathrm{H}_{\mathrm{d}}^{+} \mathrm{Ndx}}+\frac{\delta \overline{\mathrm{R}}}{\overline{\mathrm{R}}}$

In using this perturbation expression, [or equations (11) or (15)], a change in the weighting function $\mathrm{H}^{+}$leads not only to a change in the associated ratio but also in the production and removal terms of $M$. Thus, we associate the component $\delta M_{n}$ with $\delta H_{n}^{+}$, and $\delta M_{d}$ with $\delta H_{d}^{+}$. Additionally, in the critical system, there is 1 ike1y to be a further compensating perturbation to keep the system critical. Operationally, this might be a withdrawal of control rods, introduction of fresh fuel, etc. A convenient mathematical adjustment is to make a uniform change in the production operator. The choice for this compensation may itself change the ratio somewhat. If we represent the selected control compensation by a change $\delta \mathrm{M}_{0}$, then the magnitude of this correction is given by conventional perturbation theory; to remain critical, we must have, to first order,

$$
\int \mathrm{N}^{+}\left[\delta \mathrm{M}_{\mathrm{O}}+\delta \mathrm{M}_{\mathrm{n}}+\delta \mathrm{M}_{\mathrm{d}}\right] \mathrm{Ndx}=0
$$


SOLVING THE ADJOINT EOUATIONS

The equation for $\mathrm{S}^{+}$, Equation (3), may be solved by a number of techniques. The two we outline are the expansion in the eigenfunctions of the source free equation and the iteration of the source free equation. The former is sometimes convenient for analytical treatment of perturbations. The latter is easily adapted to standard reactor physics codes calculating the critical system (if the code does not already offer the option of an adjoint source in a sub-critical system) and is suitable for variational and perturbation theory.

For the first method, we require the eigenfunctions of the equations:

$$
\left[\lambda_{\mathrm{p}} \mathrm{F}-\mathrm{R}\right] \mathrm{N}_{\mathrm{p}}=0 \quad ; \quad\left[\lambda_{\mathrm{q}} \mathrm{F}^{*}-\mathrm{R}^{*}\right] \mathrm{N}_{\mathrm{q}}^{+}=0
$$

Multiplying each equation by its counterpart eigenfunction and subtracting gives

$$
\left[\lambda_{p}-\lambda_{q}\right] \int N_{q}^{+} F N_{p} d x=0 .
$$

Now the lowest modes are all of the same sign. If $F$ is a nonnegative operator (positive neutrons have positive progeny) then the integral for $\mathrm{p}=\mathrm{q}=0$ will not vanish. Therefore, in the lowest mode, the eigenvalue from the adjoint equation equals the lowest mode eigenvalue from the density equation. This is a result we had anticipated physically in saying the importance equation went "critical" at the same time as the density.

We assume the set of eigenfunctions $\mathrm{N}_{\mathrm{p}}^{+}$are sufficiently complete, and that the eigenvalues are sufficiently well behaved. (This is probably valid for diffusion theory and group models but unjustified for continuous transport theory!) Then expand $\mathrm{S}^{+}$in the set of the $\mathrm{N}_{\mathrm{p}}^{+}$.

$$
S^{+}=\sum_{p=0}^{\infty} N_{p}^{+}
$$


In principle, the summation is from the lowest mode $\left(N_{p}^{+}\right)$to infinity $\left(\mathrm{N}_{\infty}^{+}\right)$though in practice of course, we shall evaluate a limited number of terms. To determine the normalization of the $\mathrm{N}_{\mathrm{p}}^{+}$, we introduce the expansion into the equation for $\mathrm{S}^{+}$and make use of the orthogonality relation, that $\int \mathrm{N}_{q}^{+} F N_{p} d x$ vanishes for $\lambda_{p} \neq \lambda_{q}$. We have

$$
\left[\mathrm{F}^{*}-\mathrm{R}^{*}\right] \sum_{\mathrm{p}} \mathrm{N}_{\mathrm{p}}^{+}+\mathrm{H}^{+}=0 \text {. }
$$

Multiply by $\mathrm{N}_{\mathrm{q}}$ and integrate, and having replaced $\mathrm{R}^{*} \mathrm{~N}_{\mathrm{p}}^{+}$with $\lambda_{\mathrm{p}} \mathrm{F}^{*} \mathrm{~N}_{\mathrm{p}}^{+}$,

$$
\int N_{q}^{+} F N_{q} d x=\frac{\int H^{+} N_{q} d x}{\lambda q-1}
$$

This results gives us the normalization of $\mathrm{N}_{\mathrm{p}}^{+}$. Since $\lambda_{q}-1>0$ in a sub-critical system and increases with higher modes, the expansion coefficients get smaller in higher modes. In particular, if the system is critical in the lowest mode, $\lambda_{0}=1$, then the lowest mode component of the expansion is unbounded as anticipated.

The alternative solution method is by the iterative scheme. Let us define an algorithm to compute successive iterations $\stackrel{\imath}{i}+(j)$ by

$$
R *{ }^{*} N^{+}(1)=H^{+} ; R^{*} \tilde{N}+(j+1)=F * \tilde{N}(j) \quad j=1,2,3 \ldots
$$

This is the standard iterative scheme that starts from an arbitrary initial value $\mathrm{H}^{+}$to generate successive iterations, generation importances or iterated fission probabilitics. It is the counterpart of the widely used method to obtain generation densities starting from an arbitrary source. It is most common $1 y$ used to solve the source-free equation because, after sufficient iterations, the iterates converge to the exact solution of the 
critical importance equation. Furthermore, if the system is not critical, iterations of successive iterates gives the eigenvalue or effective multiplication of the system.

Let us demonstrate this convergence of the adjoint iterative scheme. We shall employ the Green's function of the production free equation, which is a solution of

$$
\mathrm{R}^{*} \mathrm{~g}^{\star}\left(\mathrm{x} / \mathrm{x}^{\prime}\right)=\delta\left(\mathrm{x}-\mathrm{x}^{\prime}\right)
$$

Let $G^{*}$ be the integral operator such that

$$
\mathrm{G}^{*} \psi^{+}=\int \mathrm{g}^{*}\left(\mathrm{x} / \mathrm{x}^{\prime}\right) \psi^{+}\left(\mathrm{x}^{\prime}\right) \mathrm{d} \mathrm{x}^{\prime}
$$

Then the adjoint eigenvalue equation can also be written as

$$
\tilde{N}_{p}^{+}=\lambda_{p} G^{*} F^{*} \tilde{N}_{p}^{+}
$$

It is also clear, that however we carry out the iterations, the algorithm consists of taking an arbitrary source term and determining the importance in a production free system. Thus, we. may write

$$
\tilde{N}^{+}(j+1)=G^{*} F^{\star}{ }_{N}^{+}+(j)
$$

Now let us again expand in the adjoint eigenfunctions, this time expanding the $j^{\text {th }}$ iterate. We have

$$
\tilde{N}^{+(j+1)}=\sum_{p} G^{*} F^{*} N_{p}^{+}=\sum_{p} \frac{N_{p}^{+}}{\lambda_{p}} .
$$

Without even determining the expansion coefficients, we see that one iteration has the effect of dividing each modal component by its eigenvalue. Since the lowest mode has the smallest eigenvalue, the higher modes are attenuated compared to the lowest or fundamental mode. After many iterations, the higher modes can be made as small as we like in comparison with the fundamental. 
Finally, we see that for sufficient iterations, we converge. Furthermore,

$$
\operatorname{Lim}_{j \rightarrow \infty} \frac{\tilde{N}^{+}(j+1)(x)}{\tilde{N}^{+}(j)(x)}=\frac{1}{\lambda_{0}}
$$

so that the converged ratio of successive iterates gives us the effective multiplication factor.

Now consider our previous expansion of the source importance, but modify it by summing over the successive adjoint iterations or generation importances. Suppose

$$
S^{+}=\sum_{j=1}^{\infty} \tilde{N}^{+}(j)
$$

In a subcritical system, $\lambda_{0}$ is larger than unity (in order to make the production term balance the removal term in the eigenvalue equation). Therefore, after many iterations, the $\tilde{N}^{+}(j)$ iterate is negligibly small, and we can expect such a summation to converge. Now we seek to show that the sum also satisfies equation for the source importance. To do this, we operate on it by $R^{*}$ :

$$
R^{*} S^{+}=\sum_{j=1}^{\infty} R^{* \sim} N^{+}(j)=H^{+}+\sum_{j=2}^{\infty} F N^{*}(j-1)=H^{+}+\sum_{j=1}^{\infty} F N^{*}{ }^{+}(j)=11^{+}+F^{*} S^{+} \text {. }
$$

This is the same as Equation (4) and gives us an alternative method of solving for $\mathrm{S}^{+}$, by accumulating successive iterations, starting from $\mathrm{H}^{+}$as our arbitrary initial estimate of the production source term.

In a critical system, the adjoint functions $\mathrm{C}_{i}^{+}$may be expressed by the same expansion in eigenfunctions. Indeed, the cquations are essentially the same except that we have deducted the lowest mode component from the source. Therefore,

$$
C^{+}=\sum_{p=1}^{\infty} N_{p}^{+} \quad p \neq 0
$$

where the normalization continues to be given by Equation (30). 
As before, a more practical solution method for most reactor systems comes from an iterative scheme. Comparison with the previous discussion suggests putting

$$
C^{+}=\sum_{j=1}^{\infty}\left[\tilde{N}^{+}(j)-\tilde{N}^{+(\infty)}\right]
$$

This clearly leads to vanishing terms for sufficient $j$ in critical systems. Let us show that this sum of residual importances in higher modes satisfies the correct equation by operating on $\mathrm{C}^{+}$with $\mathrm{R}^{*}$.

$$
\begin{aligned}
& R^{*} C^{+}=\sum_{j=1}^{\infty}\left[R^{*} N^{+}(j)-R^{*} \tilde{N}^{+}(\infty)\right]=H^{+}-F^{*} \tilde{N}^{+(\infty)}+\sum_{j=2}^{\infty} F^{*}\left[\tilde{N}^{+}(j-1)-\tilde{N}^{+(\infty)}\right] \\
& =\mathrm{H}^{+}-\mathrm{F}_{\mathrm{N}}^{*}{ }^{+}(\infty) \mathrm{F}^{*} \mathrm{C}^{+}
\end{aligned}
$$

If we identify $\mathrm{H}^{+}$, the arbitrary critical source in our iteration scheme, with the adjoint source in Equation (19) we see that $\mathrm{C}^{+}$satisfies the correct equation. Furthermore, $\tilde{\mathrm{N}}^{+}(\infty)$, the fully converged iterated solution, is the lowest mode importance solution with the 'correct normalization. Thus, the iterative scheme can be used to compute the $\mathrm{C}_{i}^{+}$in a convenient manner, starting with a zeroth iterate source equal to the weighting function of interest.

To show that $\tilde{\mathrm{N}}^{+(\infty)}$ is correctly normalized, multiply the iteration algorithm [Equation (31)] by $N$ and integrate

$$
\begin{aligned}
& \int N R^{*}{ }_{N}^{+}(1) d x=\int N H^{+} d x=\int \tilde{N}^{+(1)} R N d x=\int \tilde{N}^{+(1)} F N d x \\
& \int N R^{*} \stackrel{N}{N}+(j+1) d x=\int \tilde{N}+(j+1) R N d x=\int \tilde{N}+(j+1) F N d x \\
& =\int N F^{*} \tilde{N}+(j) d x=\int N^{+}(j) F N d x .
\end{aligned}
$$

Then, by induction,

$$
\overline{\mathrm{N}}=\int \mathrm{H}^{+} \mathrm{N} \mathrm{dx}=\int \tilde{N}^{+(\infty)} \text { FNdx } .
$$




\section{CONCLUSION}

Here are a few points regarding the implementation of the computational schemes just outlined and their application to the stationary expressions. First, the operator $M$ in diffusion theory is typically

$$
\left[\nabla \cdot \mathrm{D} \nabla-\sum^{\mathrm{a}}+x \sum^{\mathrm{f}}\right] \emptyset=0
$$

(We have not shown scattering between groups explicitly). For reasons that are related to the difficulty of securing trial functions continuous in the current (such being a condidion for the commutation of the adjoint diffusion operator), it is convenient to replace this by the operator $M$ where, for any flux and adjoint functions,

$$
\psi^{+} M_{\psi}=-\nabla \psi^{+} \cdot D \nabla \psi-\psi^{+} \sum_{\psi}^{a_{\psi}+\psi^{+}} \times \sum^{f_{\psi}} \cdot
$$

This represents the integrand appearing in the variational principle. The usual adjoint operator, however, appears in the adjoint equation used to solve or estimate for the importance.

For analogous reasons, in perturbation theory, we may not write a term in $\nabla \cdot \delta \mathrm{D} \psi$. Instead, evaluate the perturbation expression,

$$
\psi^{+} \delta \mathrm{M} \psi=-\nabla \psi^{+} \cdot \delta D \nabla \psi-\psi^{+} \delta \sum^{\mathrm{a}_{\psi}+\psi^{+} \delta\left(x \Sigma^{+}\right) \psi} \cdot
$$

When the less usual boundary conditions of diffusion theory are used, additional terms appear; the reader may refer to Reference (3). Integral operators do not require this modification, but it is required in higher order differential theories.

In computing either $\mathrm{S}^{+}$or $\mathrm{C}^{+}$iteratively, we shall not, of course, carry out an infinite number of iterations. Depending on the convergence criterion, only a few iterations may be necessary. $C^{+}$may be computed by accumulating iterates as if $S^{+}$were to be formed and, if $j$ iterates have been performed, subtracting $j$ times the jth iterate to obtain $\mathrm{C}^{+}$from $\mathrm{S}^{+}$. Often the $\mathrm{jth}$ 
iterate, as an approximation for the lowest mode importance, will be of interest in its own account.

Note that the $\mathrm{C}^{+}$solutions will be part positive, where the positive adjoint source dominates; part negative, where $\mathrm{F}^{*} \mathrm{~N}^{+}$ dominates. The code must not reject negative solutions as erroneous :

Rather than test the density or adjoint functions for convergence, we can base our convergence criterion on the ratio itself, estimating $\bar{R}^{(j)}$ after every iteration and ceasing when the convergence of the ratio itself is adequate. Use can also be made of an intercomparison of the eigenvalues obtained by comparing successive iterates of the density and adjoint functions. If, however, it is required to estimate the eigenvalue as part of the problem, this should, of course, be done using the Rayleigh quotient as a stationary functional for the effective multiplication.

$$
\bar{L}_{r}=\frac{\int \tilde{N}+(j)_{F}^{*} N+(j+1) d x}{\int \tilde{N}+(j) F F^{*}(j) d x} \rightarrow \frac{1}{\lambda_{0}}
$$

These remarks assume that the calculations of the functions are being carried on simultancously.

In calculating the $\mathrm{C}^{+}$, it may be that the system is not critical but has an effective multiplication factor $1 / \lambda_{0} \neq 1$. Then the computation would not converge. Most codes, however, automatically renormalize successive iterations. While this is an approximate correction in that it guarantees convergence, a more accurate, if a more tedious procedure, is to store successive iterates separately and correct them by multiplying by $\lambda_{0}^{j}$ after $\lambda_{0}$ has finally been determined from the last iterates. The renormalization of iterates must not be applied in computing $\mathrm{S}^{+}$ iteratively.

It is hoped that this discussion of the stationary method will enable ratios of interest in reactor physics to be computed 
in an efficient manner. The iterative algorithms for computing the various adjoint functions are basically only a modification of existing schemes. Therefore, one can have every confidence in their success. It has also been emphasized that if the program is written to take advantage of simultaneous calculations of the functions, even greater economies accrue. With physical understanding and some degree of sophistication, the stationary method applied to digital codes will be as much benefit as they have proved earlier in analytic and hand calculations. 


\section{REFERENCES}

1. L. M. Usachev. "Perturbation Theory for the Breeding Ratio and for Other Number Ratios Pertaining to Various Reactor Processes," Atomnaya Energiya, vol. 15, pp. 472-481. Trans Consultants Bureau, New York, 1964. Also in J Nucl Energy vol. A/B 18, pp. 571-583. 1964.

2. B. H. Duane. Program S; Variational Optimum Formulation, XDC-59-9-118. General Electric Co. Aircraft Nuclear Propulsion Dept., Cincinnati. 1959.

3. J. Lewins. Importance; The Adjoint Function, Pergammon Press, Oxford, England. 1965

4. A. A. Abagain, G. L. Drujinma, A. A. Dubinin, S. M. Zaritsky, V. V. Orlov, Y. A. Pubkov, A. P. Suvarov, L. M. Usachev, and R. P. Foderenko. "Some New Aspects of Using Adjoint Functions and Perturbation Theory in Reactor and Shielding Calculations," International Conference on Peaceful Uses of Atomic Energy, Geneva, Switzerland, p-364, 1964. (To be published, United Nations, New York) 
APPENDIX A 


\section{APPENDIX A}

TABLE OF STATIONARY EXPRESSIONS FOR THE RATIO

$$
\overline{\mathrm{R}}=\int \mathrm{H}_{\mathrm{n}}^{+} \mathrm{Ndx} / \int_{\mathrm{d}^{+}}^{+} \mathrm{Ndx}
$$

Subcritical with Source

$$
\mathrm{MN}+\mathrm{S}=0 ; \mathrm{M}^{*} \mathrm{~S}^{+}+\mathrm{H}^{+}=0 ; \mathrm{H}^{+}=\frac{\mathrm{H}_{\mathrm{n}}^{+}}{\int \mathrm{H}_{\mathrm{d}}^{+} \mathrm{Ndx}}-\frac{\overline{\mathrm{R}}}{\int \mathrm{H}_{\mathrm{d}}^{+}} \frac{\mathrm{H}_{\mathrm{d}}^{+} \mathrm{Nd}}{}
$$

1. $\overline{\mathrm{L}}_{\mathrm{S}}=\int\left[\mathrm{H}^{+} \mathrm{N}+\mathrm{S}^{+} \mathrm{MN}+\mathrm{S}^{+} \mathrm{S}\right] \mathrm{dx} \rightarrow \overline{\mathrm{R}}$

$$
\delta \overline{\mathrm{L}}_{\mathrm{S}}\left(\delta \mathrm{H}^{+}, \delta \mathrm{M}, \delta \mathrm{S}\right)=\int\left[\delta \mathrm{I}^{+} \mathrm{N}+\mathrm{S}^{+} \delta \mathrm{MN}+\mathrm{S}^{+} \delta \mathrm{S}\right] \mathrm{dx} \rightarrow \delta \overrightarrow{\mathrm{R}}
$$

where

$$
\delta H^{+}=\frac{\delta H_{n}^{+}}{\int H_{d}^{+} N d x}-\bar{R} \frac{\delta H_{d}^{+}}{\int H_{d}^{+} N d x}
$$

2. $\overline{\mathrm{L}}=\frac{\overline{\mathrm{L}}_{\mathrm{n}}}{\overline{\mathrm{L}}_{\mathrm{d}}}+\overline{\mathrm{R}}$

where

$$
\begin{aligned}
& \bar{L}_{i}=\int\left[H_{i}^{+} N+S_{i}^{+} M i+S_{i}^{+} S\right] d x \quad i=n, d \\
& \frac{\delta \bar{L}\left(\delta H^{+}, \delta M, \delta S\right)}{\bar{R}}=\frac{\int\left[\delta H_{n}^{+} N+S_{n}^{+} \delta M N-S_{n}^{+} \delta S\right] d x}{\int H_{n}^{+} N d x} \\
& -\frac{\int\left[\delta H_{d}^{+} N+S_{d}^{+} \delta M N+S_{d}^{+} \delta S\right] d x}{\int H_{d}^{+} N d x}
\end{aligned}
$$




\section{Critical Systems}

$$
M N=0 ; M^{*} C_{i}^{+}+H_{i}^{+}-F^{*} N_{i}^{+}=0 ; M^{*} N_{i}^{+}=0 \quad i=n, d
$$

3. $\bar{L}_{\mathrm{C}}=\frac{\int\left[\mathrm{H}_{\mathrm{n}}^{+}+\mathrm{C}_{\mathrm{n}}^{+} \mathrm{M}\right] N \mathrm{dx}}{\int\left[\mathrm{H}_{\mathrm{d}}^{+}+\mathrm{C}_{\mathrm{d}}^{+} \mathrm{M}\right] N \mathrm{~N} \mathrm{x}} \rightarrow \overline{\mathrm{R}}$

$$
\frac{\delta \overline{\mathrm{L}}_{\mathrm{C}}\left(\delta \mathrm{M}^{+}, \delta \mathrm{M}\right)}{\overline{\mathrm{R}}}=\frac{\int\left[\delta \mathrm{H}_{\mathrm{n}}^{+}+\mathrm{C}_{\mathrm{n}}^{+} \delta \mathrm{M}\right] \mathrm{Ndx}}{\int \mathrm{H}_{\mathrm{n}}^{+} N \mathrm{dx}}-\frac{\int\left[\delta \mathrm{H}_{\mathrm{d}}^{+}+\mathrm{C}_{\mathrm{d}}^{+} \delta \mathrm{M}\right] N \mathrm{dx}}{\int \mathrm{H}_{\mathrm{d}}^{+} N \mathrm{dx}}
$$

4. $\bar{L}_{r}=\frac{\int_{N}+(j) F N(j+1) d x}{\sqrt{N+(j)} F N^{(j)} d x} \rightarrow \frac{1}{\lambda_{O}}$

$$
\delta \overline{\mathrm{L}}_{\mathrm{r}}(\delta M)=\frac{\int N^{+} \delta M N d x}{\int N^{+} \text {FNdx }} \rightarrow \delta\left(\frac{1}{\lambda_{0}}\right)
$$




\section{$\underline{\text { ONSITE DISTRIBUTION }}$}

\section{Copy Number}

Pacific Northwest Laboratory

D. G. Albertson

C. A. Bennett

C. L. Bennett

R. A. Bennett

S. R. Bierman

C. L. Brown

W. W. Brown

S. H. Bush

G. J. Busse1man

$J$. J. Cadwe 11

J. L. Carter

L. L. Carter

D. E. Christensen

R. G. Clark

E. D. Clayton

R. E. Dah 1

L. C. Davenport

F. G. Daws on

D. R. de Halas

R. F. Dickerson

B. H. Duane

M. R. Egan

G. W. R. Endres

E. A. Eschbach

S. L. Fawcett

J. R. Fishbaugher

D. G. Foster

H. A. Fowler

J. J. Fuquay

A. G. Gibbs

D. W. Glasgow

C. E. Haines

R. W. Hardie

O. K. Harling

R. A. Harris

H. Harty

R. A. Harvey

C. M. Heeb

R. E. He ineman

H. L. Henry

N. A. Hi 11

R. J. Hoch

P. L. Hofmann 
ONSITE DISTRIBUTION (Contd.)

\section{Copy Number}

\begin{tabular}{|c|c|c|}
\hline 44 & R. $\mathrm{H}$. & Holeman \\
\hline 45 & R. M. & Humes \\
\hline 46 & U. P. & Jenquin \\
\hline 47 & R. L. & Junkins \\
\hline 48 & $\mathrm{E}$. & Kelley, Jr. \\
\hline 49 & D. A. & Kottwitz \\
\hline 50 & J. & Kutcher \\
\hline 51 & C. R. & Lagergren \\
\hline 52 & D. D. & Lanning \\
\hline 53 & $\mathrm{~J} . \mathrm{H}$. & Lauby \\
\hline 54 & $B$. & Leonard, Jr. \\
\hline 55 & W. & Lewis \\
\hline 56 & $\mathrm{R}$. & Liikala \\
\hline-61 & C. & Lindenme ie $r$ \\
\hline 62 & W. & Little \\
\hline 63 & $\mathrm{R}$. & Lloyd \\
\hline 64 & L. & Maas \\
\hline 65 & D. & Marr \\
\hline 66 & $\mathrm{R}$. & Matsen \\
\hline 67 & D. & Matsumoto \\
\hline 68 & R. & Nightingale \\
\hline 69 & $\mathrm{~L}$. & Page \\
\hline 70 & H. M. & Parker \\
\hline 71 & R. S . & Paul \\
\hline 72 & R. E. & Peterson \\
\hline 73 & W. & Reardon \\
\hline 74 & $\mathrm{~J}$. & Regimba 1 \\
\hline 75 & C. R. & Richey \\
\hline 76 & W. & Roesch \\
\hline 77 & J. T. & Russe 11 \\
\hline 78 & J. E. & Schlosser \\
\hline 79 & R. E. & Schenter \\
\hline 80 & $\mathrm{~L}$. & Schmid \\
\hline 81 & D. R. & Skeen \\
\hline 82 & J. D. & Smith \\
\hline 83 & R. B & Smith \\
\hline 84 & $\mathrm{R}$. & Smith \\
\hline 85 & K. B. & Stewart \\
\hline 86 & W. P. & Stinson \\
\hline 87 & $\mathrm{~J} . \mathrm{J}$ & Stoffels \\
\hline 88 & D. $\mathrm{H}$ & Thomsen \\
\hline 89 & T. B. & Thornbury \\
\hline 90 & C. R. & Tipton, Jr. \\
\hline 91 & V. 0 & Uotinen \\
\hline 92 & E. E . & Voil and \\
\hline 93 & M. T. & Wa11ing, Jr. \\
\hline 94 & J. D. & White \\
\hline
\end{tabular}




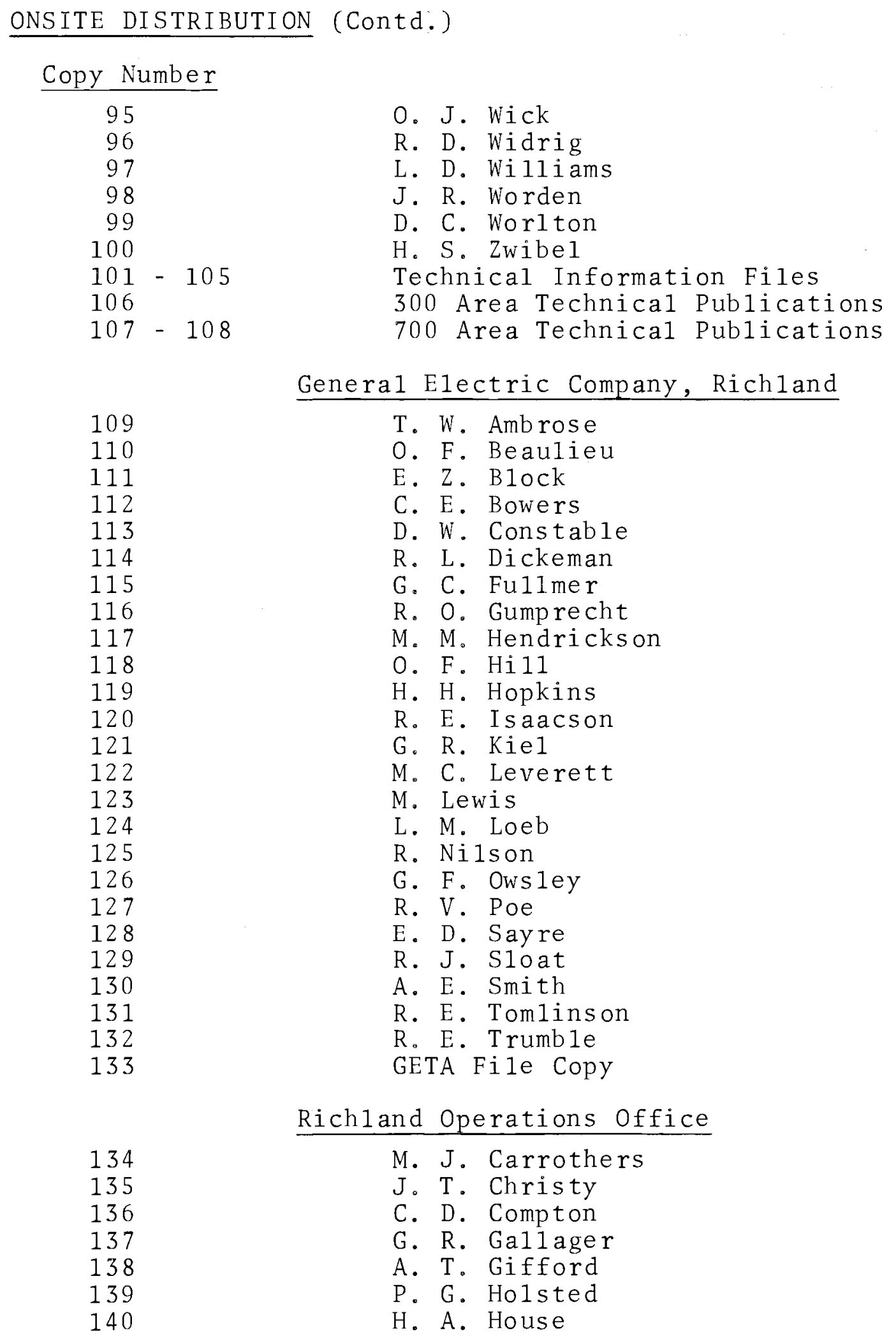


ONSITE DISTRIBUTION (Contd.)

Copy Number
141
142
143
144
145
146
147
J. M. Musser
R. L. Plum
R. G. Rader
M. J. Rasmussen
M. R. Schneller
R. K. Sharp
Technical Information Library

\section{OFFSITE DISTRIBUTION (SPECIAL)}

No. of Copies

1

1

4

1

11
Air Force Surgeon General

Albuquerque Operations Office

Argonne National Laboratory

Reactor Physics Constants Center

Atomic Bomb Casualty Commission

Atomic Energy Commission, Washington

Attn: Assistant Director for Civilian Reactors, DRD (1)

R. P. Baranowski, Director, Division of Production (1)

Chief, Physics Branch, DRD (1)

P. B. Hemmig, Physics Branch, DRD

K. G. Kolstad, Assistant Director for Physics and Mathematics Programs (1)

C. D. Luke, Division of Licensing and Regulations (1)

R. HI. Odegaarden, Division of Licensing and Regulations (1)

J. L. Schwennesen, Assistant Director, Reactor Products, Division of Production (1)

E. E. Sinclair, Assistant Director for Advanced Reactor Technology

Water Reactor Branch, DRD (2)

AEC Scientific Representative Argentina

AEC Scientific Representative, Relgium

Bechiel Corporation

Bureau of Naval Weapons 


\section{OFFSITE DISTRIBUTION (SPECIAL) (Contd.)}

No. of Copies

1

1

2

2

1

1

1

1

1

1

1

1

1

1

1

1

1

1

1
California Institute of Technology

Attn: H. Lurie, Engineering Division

Columbia University (NYO-187)

Corne11 University, Ithaca, N。Y.

Attn: R. T. Cuykenda11, Engineering Physics

R. R. Witson, Physics Department

Duke University, Durham, N. C.

Attn: H. W. Newson, Physics Department

W. J. Seeley, School of Engineering

Edgewood Arsenal

Federal Aviation Agency

Institute of Atomic Physics

Applied Radioactivity Laboratory

Bucuresti, CP 35, Rumania

Attn: Ing. E. Gaspar

Inter-American Institute of Agricultural Sciences

Japan Atomic Energy Research Institute (JAERI)

Tokai-mura, Naka-gun, Ibaraki-ken, Japan

Attn: Hajime Sakata

Kansas State University, Manhattan, Kansas

Attn: W. R. Kime1, Nuclear Engineering

Mallinckrodt Chemical Works

Manhattan College, Riverdale, New York, N. Y. Attn: Brother Gabriel Kane

Massachusetts Institute of Technology

Attn: Prof. Irving Kaplan

New York University

Attn: Lyle Borst, Physics Department

North Carolina State College

Attn: R. L. Murray

Nuclear Utility Services

Office of General Inspector

Office of the Chief of Engineers

Puerto Rico Water Sources Authority 


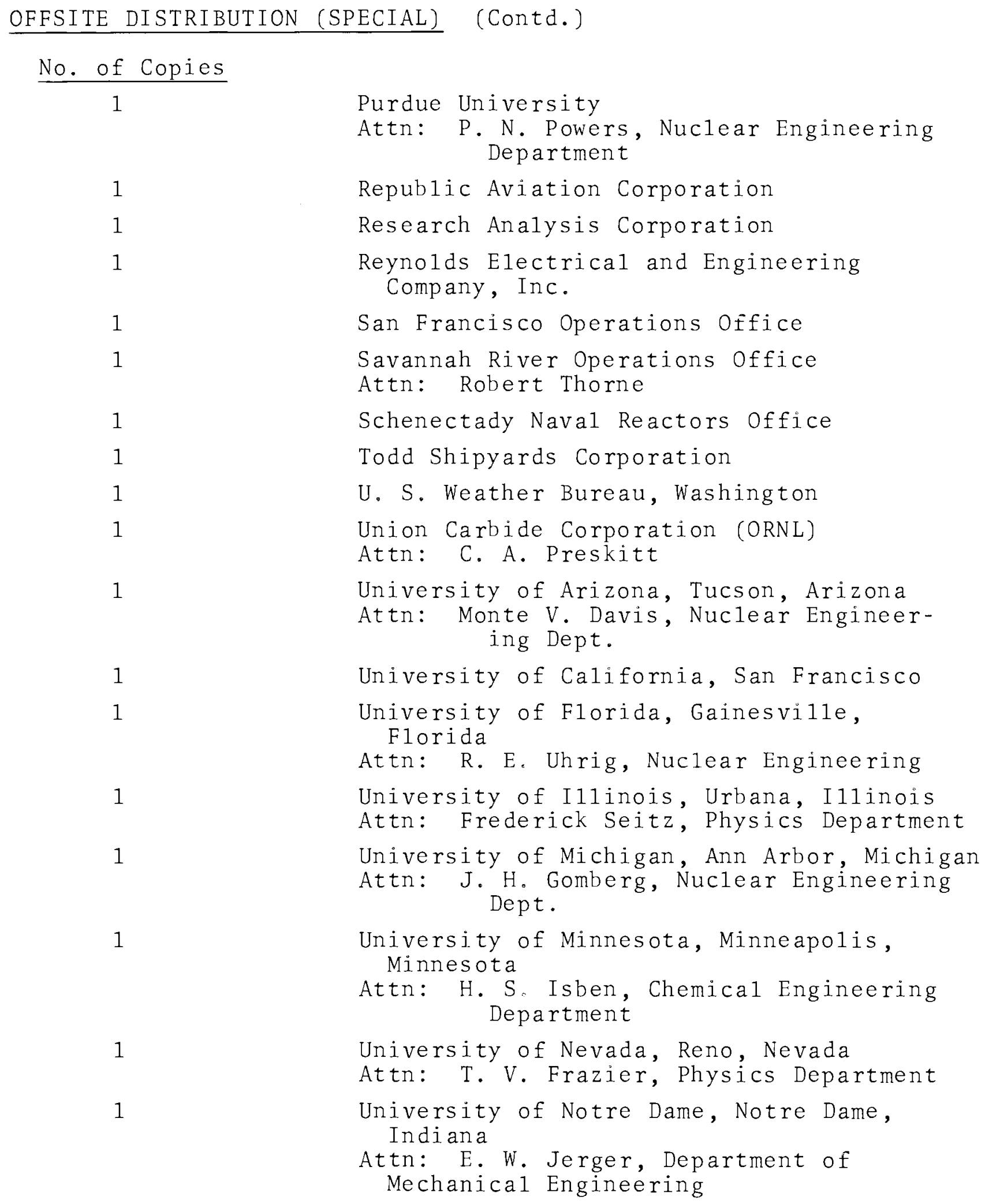




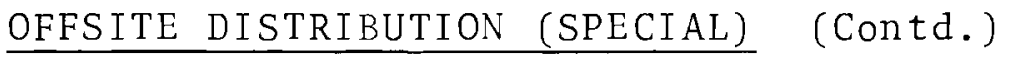 \\ No. of Copies \\ 1 \\ University of Oregon, Eugene, Oregon \\ Attn: J. L. Powe11, Physics Department \\ 2 \\ University of Tennessee, Knoxville, \\ Tennessee \\ Attn: A. H. Nielsen, Physics Department \\ P. F. Pasqua, Nuclear Engineering \\ Department \\ University of Toledo, Toledo, Ohio \\ Attn: J. J. Turin \\ University of Washington, Seattle, \\ Washington \\ Attn: A. L. Babb, Chemical Engineering \\ Department (1) \\ J. Lewins, Nuclear Engineering \\ Department (20) \\ University of Wisconsin, Madison 6 , \\ Wis cons in \\ Attn: M. W. Carbon, Nuclear Engineering \\ Committee \\ Virginia Polytechnic Institute, Blacksburg, \\ Virginia \\ Attn: Andrew Robeson, Physics Department \\ Washington State University, Pullman, \\ Washington \\ Attn: J.P. Spielman, College \\ of Engineering
}




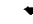

